\title{
Paper
}

\section{Floating Autostereoscopic 3D Projection Display with High Light Efficiency and Wide Viewing Depth using Anisotropic Light Diffuser}

\author{
Takahiro Ishinabe $^{\dagger 1}$ (member), Tohru Kawakami ${ }^{\dagger 2}$ (member), Masahiro Nishizawa ${ }^{\dagger 2}$, \\ Hideo Fujikake ${ }^{\dagger 1}$ (member), Tatsuo Uchida ${ }^{\dagger 3}$ (honorary member)
}

\begin{abstract}
We improved light efficiency and viewing zone depth for high-resolution floating autostereoscopic 3D display based on the iris-plane dividing technology. Newly developed light diffusion film and optical system sent almost all image light to the viewer and realize high light efficiency and uniformity. In addition, stacked liquid crystal optical shutters achieved control of the viewing zone and realized 3D images without crosstalk noise in the real space with wide depth of the viewing zone. Our floating 3D display system allows glassless and provides high-resolution 3D images with no crosstalk between left and right viewing zones. Therefore, this 3D display system is promising for the future high-presence interactive communication systems.
\end{abstract}

Keywords: Floating autostereoscopic display, Iris-plane dividing technology, Wide viewing zone depth, High light efficiency, Lihgt diffusion film, High resolution.

\section{Introduction}

Three-dimensional (3D) displays have recently attracted much interest for the next generation of display technology. High-quality 3D displays would have a significant impact on new display applications, including medical imaging and digital signage, as well as on television, cinema, and video games. In addition, if we can realize a floating $3 \mathrm{D}$ display which creates highquality $3 \mathrm{D}$ images in the real space regardless of the viewing distance, we can achieve a highly-realistic communication system using floating 3D images.

However, traditional 3D displays require the use of special glasses to achieve a $3 \mathrm{D}$ effect, and autostereoscopic 3D displays using parallax barrier or lenticular lens also suffer from low resolution, low light efficiency, lower image quality due to the cross-talk between the left and right images ${ }^{1) 2}$, and limited viewing zone. Therefore it has been difficult to realize a high quality floating 3D display based on the traditional $3 \mathrm{D}$ display systems and creation of floating

Received June 10, 2013; Revised September 4, 2013; Accepted October 17, 2013 $\dagger 1$ Department of Electronics, Graduate School of Engineering, Tohoku University (Sendai, Japan)

$† 2$ New Industry Creation Hatchery Center, Tohoku University (Sendai, Japan)

$\dagger 3$ Sendai National College of Technology (Sendai, Japan) autostereoscopic 3D display that has high resolution, high light efficiency, wide depth of the viewing zone, no cross-talk and requires no special glasses is one of the most important challenges in the development of highly realistic display systems.

We have focused our attention on the 3D display based on the iris-plane-dividing technology ${ }^{3)-11)}$ to achieve a high-resolution floating autostereoscopic 3D display and investigated an improvement in the light efficiency and viewing zone ${ }^{12)}$. In this paper, we discuss the design of an optical configuration and a light diffusion film and confirm its validity through the fabrication of a prototype system.

\section{Problems of Autostereoscopic 3D Display Based on the Iris-plane Dividing Technology}

The iris plane is a plane in an optical system that contains all information about an image and we can control the orientation of a displayed image by controlling the position of the light passing through the iris plane and using an additional lens. Fig. 1 (a) and (b) show the principle of the autostereoscopic 3D display using a liquid crystal (LC) optical shutter dividing the iris-plane for the left and right eyes, respectively.

The principal plane of Lens 1 is the iris-plane. The LC optical shutter dividing the iris-plane is located in this 


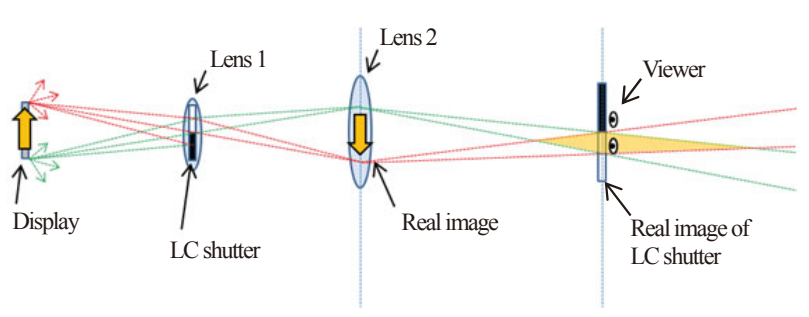

(a)

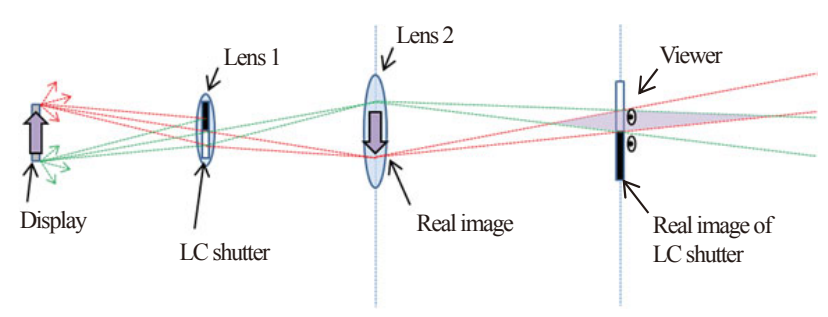

(b)

Fig.1 Principle of the 3D display based on the iris-plane dividing technology for the (a) left and (b) right eyes.

plane. The LC optical shutter controls the position of the light passing through the iris plane. Lens 2 is located where the displayed image is formed by Lens 1 . The images projected by the display passing through Lens 1 are formed at the position of Lens 2 , and are transmitted to the viewer by Lens 2. The image of the LC optical shutter is formed at the viewer's location by Lens 2 . Therefore, if the display shows the images for the right eye while the LC optical shutter closes the right half of the shutter, the displayed image is sent only to the right eye (see Fig. 1 (b)). As a result, viewers can see each of stereoscopic images with one of the eyes without special glasses by sequentially forming the left and right images or by using orthogonally polarized left and right images through the activated optical shutter in the iris plane according to the viewer's eye positions.

Based on this principle, we can realize a floating autostereoscopic 3D display by removing Lens 1 from the previous optical configuration shown in Fig.1. In this system, both the images of the LC optical shutter and the display images are generated in real space by Lens 2 13)14) (see Fig. 2).

However, this floating 3D system has the problems of the low light efficiency and narrow viewing zone depth, because the light for displayed image was diffused to wide angle range by the display device and only the light passing through the optical shutter was used to create the $3 \mathrm{D}$ images, and the optimum viewing distance imposes the same limitations as in traditional autostereoscopic 3D display systems. These problems become serious when floating images are viewed at short range, where the effect of binocular parallax is

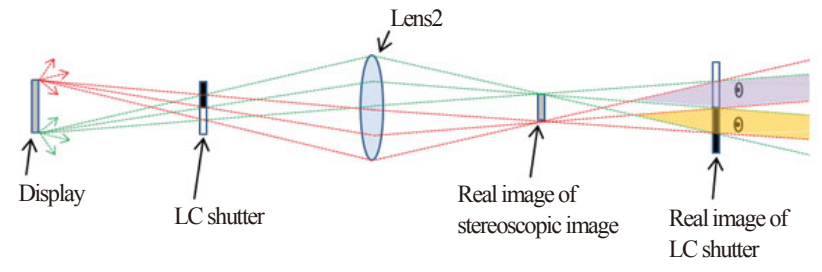

Fig.2 Configuration of the floating autostereoscopic 3D display.

important. In general, the effect of binocular parallax depends on the viewing distance, and hence a 3D display should have a wide viewing zone depth and the capability to change parallax images according to the viewing distance. Therefore, improvement of light efficiency and viewing zone depth is important for realization of high quality floating $3 \mathrm{D}$ display systems.

\section{Floating Autostereoscopic 3D Projection Display with High Light Efficiency and Wide Viewing Zone Depth}

In order to improve light efficiency, we used projector and light diffusion film and optimized its diffusion angle to send the almost all image light to the viewers. In addition, we used several LC shutter and controlled the position of real image of LC shutter to control the viewing zone depth according to the viewer's position. Fig. 3 shows the optical system of our floating 3D projection display which is composed of two micro laser projectors with orthogonal different linear polarization, polarizing beam splitter (PBS), two Fresnel lenses (Lens1 and 2), light diffusion film, LC cells, polarizer and glass lens.

We used two micro laser projectors for left and right images respectively, so we can realize high contrast and high resolution 3D images without loss of the resolution of display device and these are located at the focal point of Fresnel lens1, so the light for the display image becomes parallel after passing through the Fresnel lens1. As a result, all image lights enter into the light diffusion film.

The Fresnel lens 2 changes the direction of the principal ray of the diffused light, pointing it in the direction of its focal point. Since the diffusion angle of the front diffusion film is designed to be extremely narrow, we can send the almost all image light from projector to the viewers and realize high light efficiency. Here, the dashed triangle in Fig. 3 is the area that contains all information about an image and reserved for the optical shutter and named Iris-area. Several LC cells and single polarizer are located in this area. The LC 


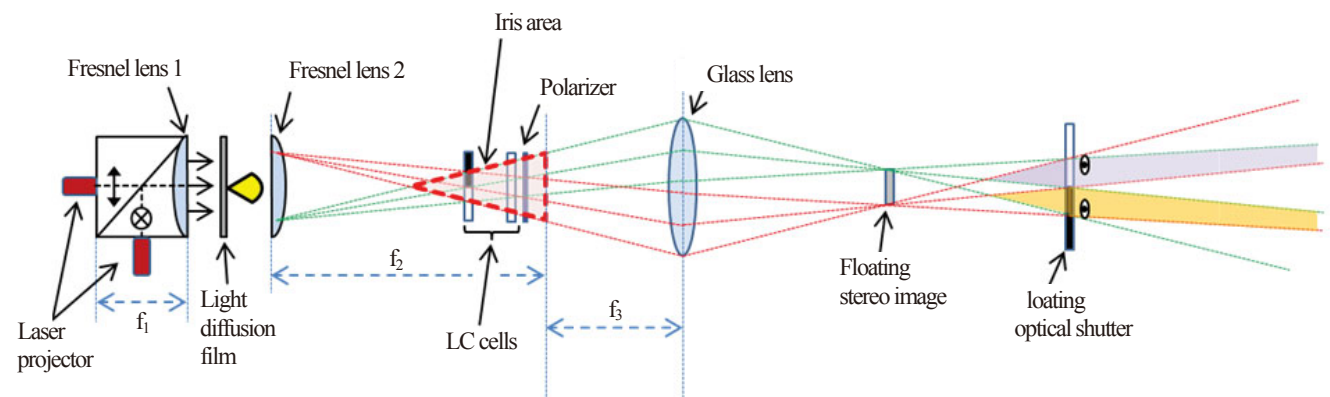

Fig.3 Floating Autostereoscopic 3D display using two projectors, light diffusion film and Fresnel lens. $\mathrm{f}_{1}, \mathrm{f}_{2}$ and $\mathrm{f}_{3}$ are focal length of Fresnel lens 1 , 2 and Glass lens.

cells rotate the polarization state of image light and work as an optical shutter in combination with a single polarizer. So we can electrically control the viewing zone discretely in the direction of the depth without any mechanical parts by changing the position of activated LC cell and its floating image according to the viewer's position. We used only one polarizer located just in front of the glass lens, so light efficiency do not degraded even for the use of several LC cells.

The glass lens is formed real images of the stereoscopic image on the light diffusion film and the LC optical shutter in the real space respectively. As a result, we can realize high resolution floating $3 \mathrm{D}$ images in the real space with a high light efficiency.

\subsection{Design of Light Diffusion Film}

A light diffusion film is an important component of our optical system. If the light from projector is not diffused, both Iris-area and viewing zone are formed at one point in the space. In this case observer cannot view images with left and right eyes.

In our display system, we used two orthogonal linear polarizations for left and right images, so light diffusion film must be capable of maintaining the polarization to eliminate a crosstalk. In addition, the light diffusion film must also be capable of controlling the distribution of the diffused light to send the all image light to the views and achieve high light efficiency. In addition, diffusion film should have a top-hat light distribution to achieve high luminance uniformity at the viewer's position. To achieve these characteristics, we adopted a light diffusion film that has an internal refractive index layer structure and optimized its diffusion characteristics ${ }^{15)-17)}$.

The light diffusion film was synthesized by the UV polymerization of acrylates. Two acrylate monomers with differing refractive indices were mixed and copolymerized by using directional UV LEDs. We found that the internal layer structure was formed by exploiting the photopolymerization-induced phase separation effect and
Table 1 Light source shape and diffused light distribution of the new light diffusion film.

\begin{tabular}{|c|c|c|}
\hline & Light Source & Distribution shape \\
\hline Isotropic & (c) & \\
\hline $1 \mathrm{D}$ & $\begin{array}{l}9 \\
0 \\
0 \\
0\end{array}$ & \\
\hline Square & 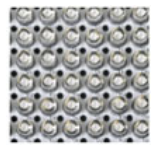 & \\
\hline Hexagonal & 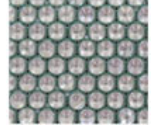 & \\
\hline
\end{tabular}

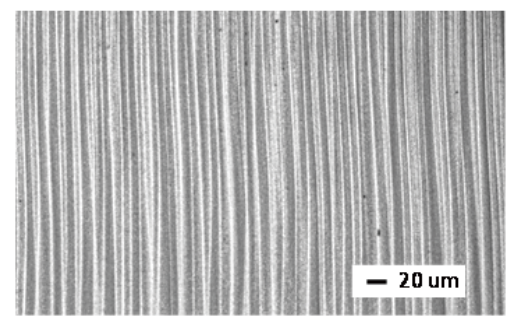

Fig.4 Internal layer structure of light diffusion film.

distribution pattern of the layer structure and diffused light corresponded to the shapes of the light sources used in the UV curing. Based on this principle, we synthesized a light diffusion film that diffuses transmitted light into square or hexagonal patterns by arranging the directional UV LEDs in tetragonally or hexagonally symmetric arrays (see Table 1).

The diffusion film consists of alternating layers with differing refractive indices. The layer pitch is $2-5$ microns, and the anisotropic light diffusion is based on diffraction by the alternating layer structure (see Fig. 4).

Fig. 5 and Fig. 6 respectively shows the distribution 


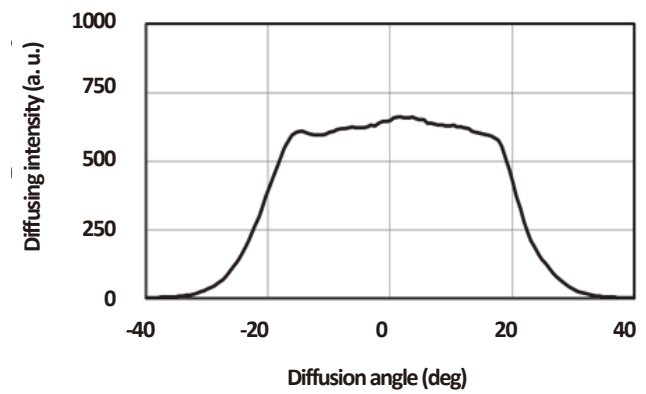

Fig.5 Diffused light distribution of newly developed light diffusion film with an internal refractive index layer structure.

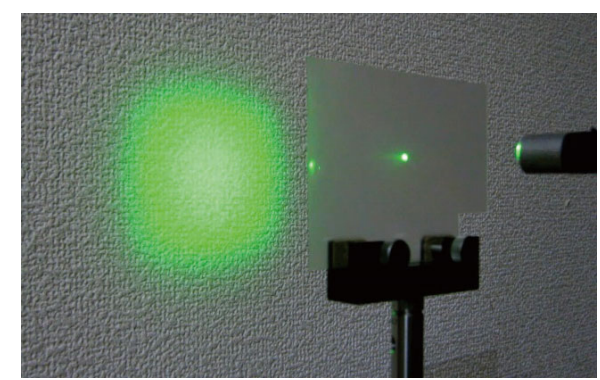

Fig.6 Square shaped light distribution of the newly developed light diffusion film.

of diffused light using our new light diffusion film optimized for floating autostereoscopic 3D display and having a square shaped light distribution. These fig.s show that the diffusing intensity is uniform, and has a top-hat distribution in the angular range of \pm 15 degrees.

A screen gain compared to the Lambiertian type screen is expressed as following equation,

$$
\mathbf{G}=\frac{\pi}{2 \sin ^{2} \theta}
$$

where $\theta$ is a diffusion angle of the light diffusion film. Diffusion angle of our diffusion film is 15degrees and a screen gain is $G=23.4$. As a result, we confirmed that our diffusion film realizes a high optical efficiency and high spatial and angular luminance uniformity at viewer positions.

Next, we measured the polarization maintenance of our light diffusion film. We set the light diffusion film between parallel and crossed polarizers and measured the light intensity between these two states. The contrast ratio of polarizer is over 2000:1 and this value is degraded by the depolarization of light diffusion film. Measured result is 650:1 and this result shows that the crosstalk ratio of our display system is $0.15 \%$. This value is extremely small compared to the traditional autostereoscopic displays.

Fig. 7 shows an image on the diffusion film though the polarizing filter.

We also measured the resolution of light diffusion film

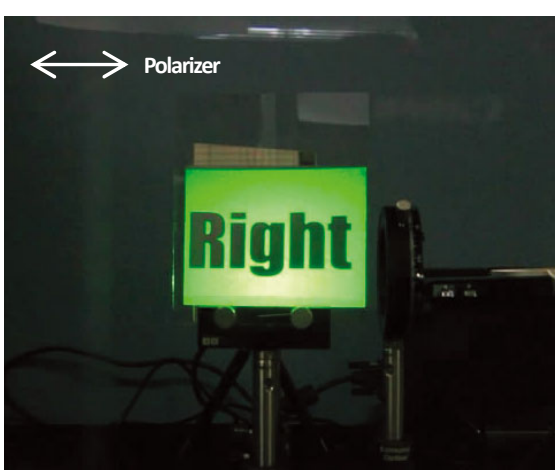

(a)

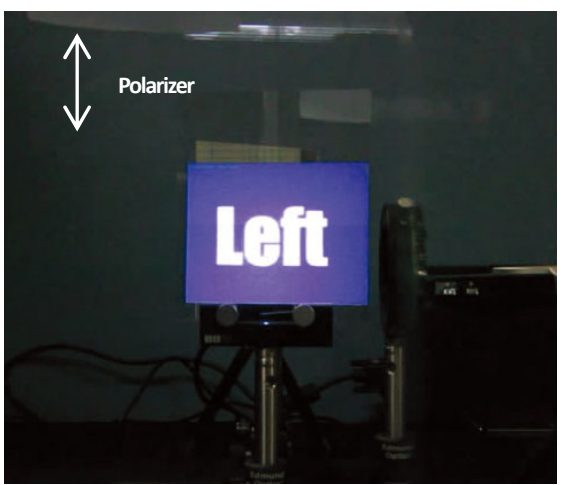

(b)

Fig.7 Image on the diffusion film though the polarizing filter.

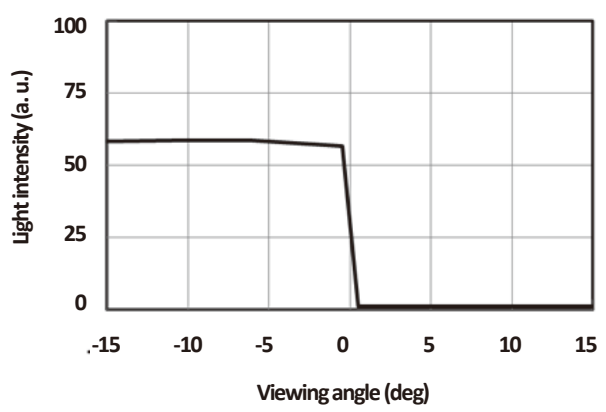

Fig.8 Luminance of 3D display system when scanning horizontally across the viewing zones.

by using the resolution chart. As a result, we confirmed that the resolution of our diffusion film is $25 \mathrm{lp} / \mathrm{mm}$ and our floating autostereoscopic 3D display can achieve high resolution $3 \mathrm{D}$ images without crosstalk noise.

Fig. 8 shows the measurement result of the luminance scanning horizontally across the viewing zones. Here, the image for the right eye was in the black state and the image for the left eye was in the white state. The luminance was uniform in the viewing zone and changed steeply at the boundary between the left and right viewing zones.

Fig. 9 shows the floating images for left and right eyes, respectively. We confirmed that our floating autostereoscopic 3D display successfully produced stereoscopic images without cross-talk noise and with 


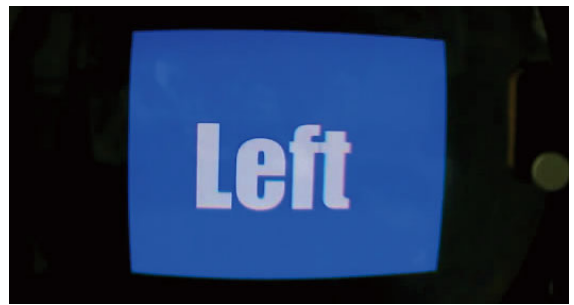

(a)

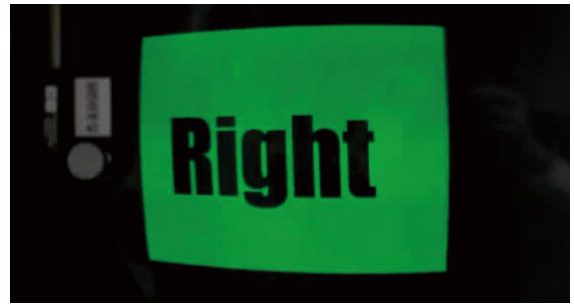

(b)

Fig.9 Floating images for (a) left and (b) right eyes.

high resolution and high luminance uniformity. However, we also found that the floating image was distorted by the glass lens.

\subsection{Depth Control for the Viewing Zone by the Stacked LC Cells}

The liquid crystal shutter dividing the iris plane is generated in real space by the glass lens, therefore the viewing zone of this system depends on the position of the liquid crystal cells in the iris area. Accordingly, we have placed several liquid crystal cells in this area. We can discretely control the viewing zone in the depth direction by purely electrical means (without any mechanical parts), by changing the position of the activated liquid crystal cell according to the viewer's position and realize wide viewing zone.

Fig. 10 shows the shift of the viewing zone toward the position of the liquid crystal cell. The focal length of the Fresnel lens 2 and glass lens were $\mathrm{f}_{2}=400 \mathrm{~mm}$ and $\mathrm{f}_{3}=$ $200 \mathrm{~mm}$, respectively. The width of the light diffusion film was $100 \mathrm{~mm}$, the size of the glass lens was $200 \mathrm{~mm}$ and the distance between light diffusion film and glass lens was $600 \mathrm{~mm}$. The LC cells should be located farther away than focal length of glass lens to form the floating image of optical shutter and the distance from glass lens were $250 \mathrm{~mm}$ and $350 \mathrm{~mm}$. Polarizer was located 200 $\mathrm{mm}$ away from the glass lens. The floating stereo image was generated $310 \mathrm{~mm}$ away from glass lens.

It is seen from Fig. 10 that we can control the viewing zone by the position of the LC cell. However, we also found that the intensity of the light passing through the LC cell and viewing area decreases for small visual distances. This problem is caused by the small width of the iris area for the small visual distance due to the

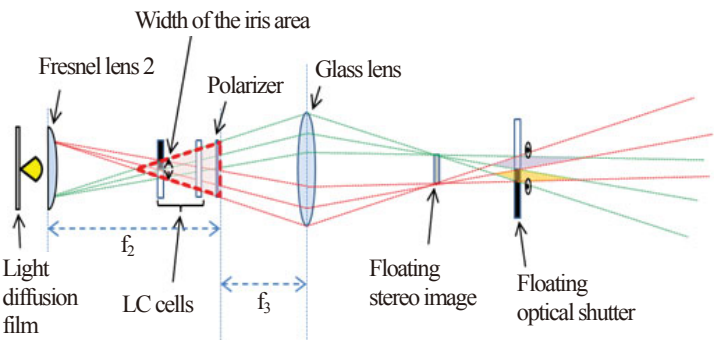

(a)

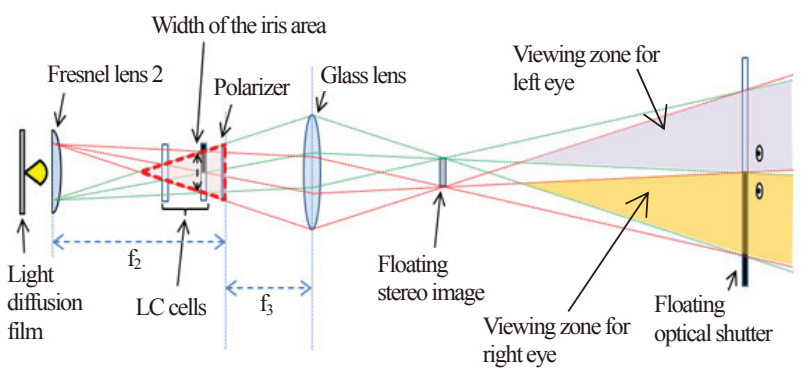

(b)

Fig.10 Depth control for the observation area by stacked LC Cells for, (a) small distance (500 $\mathrm{mm}$ from glass lens), (b) large distance (1000 mm from glass lens).

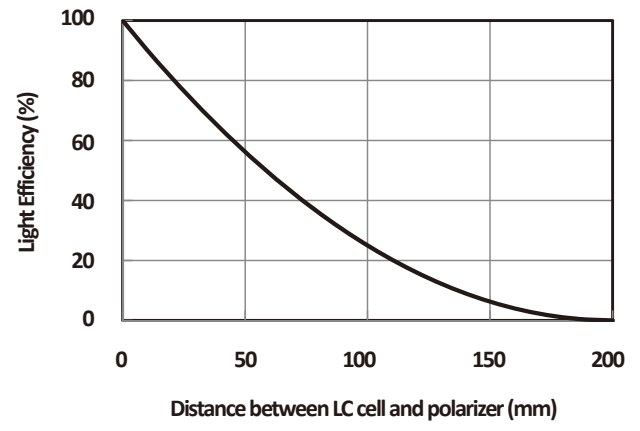

Fig.11 Change in the light efficiency with a distance between LC cell and polarizer.

triangular shape of iris area and becomes serious when viewers see floating images at short range (see Fig. 11).

To solve this problem, the iris area must be rectangular in shape, and the intensity of the light passing through the LC cell should be constant regardless of the position of LC cell. Therefore, we have modified an optical system by using two lenses and Fig. 12 shows the configuration of this system.

Lens 1 , whose focal length is $\mathrm{f}_{4}$, is located at a distance $\mathrm{f}_{4}$ from the light diffusion film. The light for the display image is collimated by Lens 1 and passes through liquid crystal cells. The displayed image is generated in real space at a distance $f_{3}$ from glass lens, which is the focal length of glass lens. The magnification ratio of displayed image was expressed as following equation.

$$
\mathrm{m}=\frac{f_{3}}{f_{4}}
$$




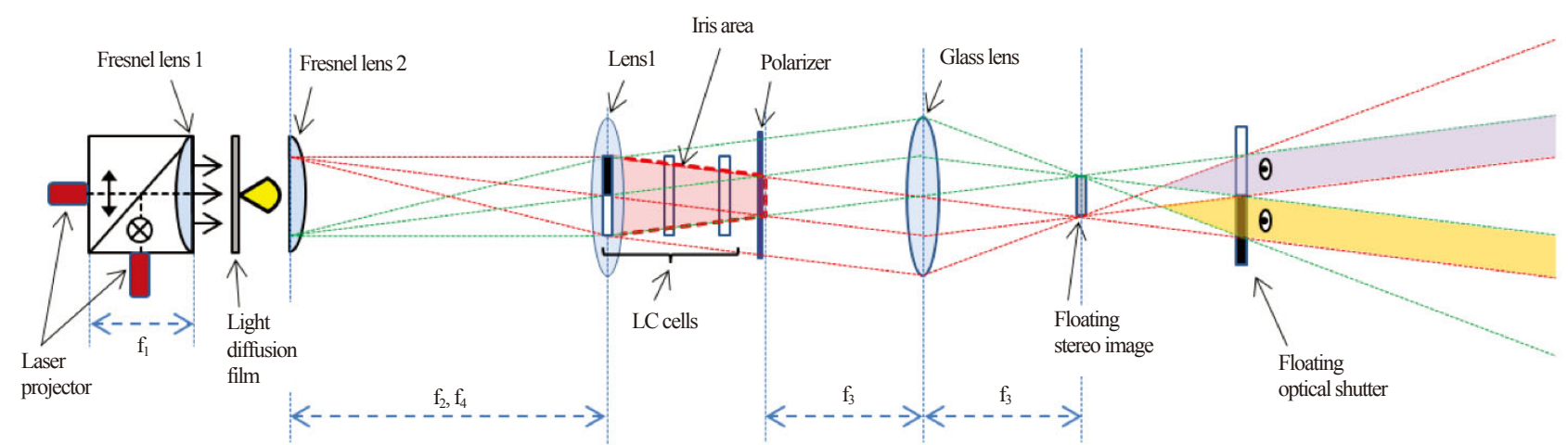

Fig. 12 Floating Autostereoscopic 3D display with a high light efficiency and a wide depth of the viewing zone. $f_{1}, f_{2}, f_{3}$ and $f_{4}$ are focal length of Fresnel lens 1, 2, Glass lens and Lens1.

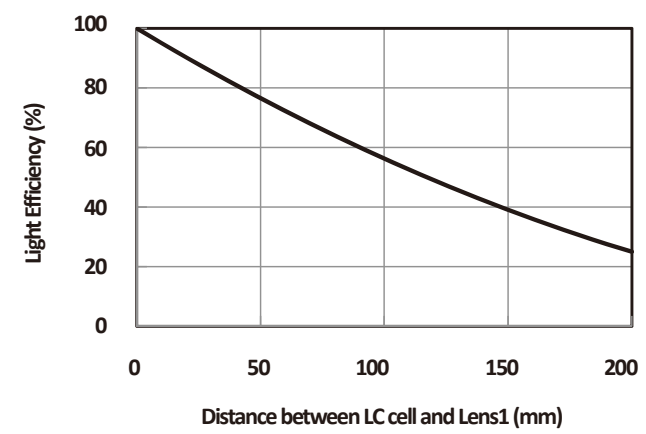

Fig.13 Change in the light efficiency with a distance between LC cell and Lens1.

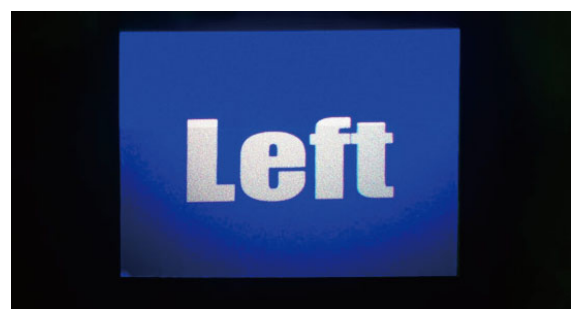

(a)

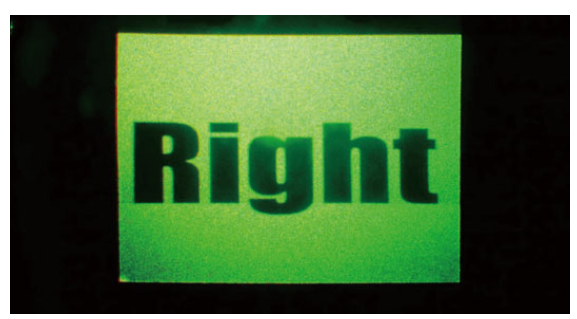

(b)

Fig.14 Floating images for (a) left and (b) right eyes.

In this situation, the iris area becomes trapezoidal in shape and the degradation of light intensity becomes relatively small toward the position of the liquid crystal cell and light efficiency becomes maximum in case of small visual distance. Fig. 13 shows the change in the light efficiency in our new optical system and we confirmed our display system has high light efficiency over a wide viewing zone depth.

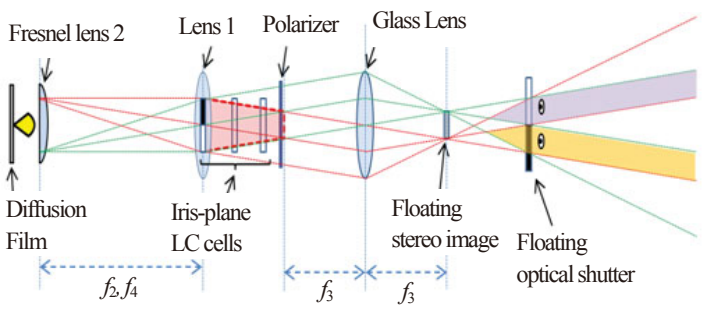

(a)

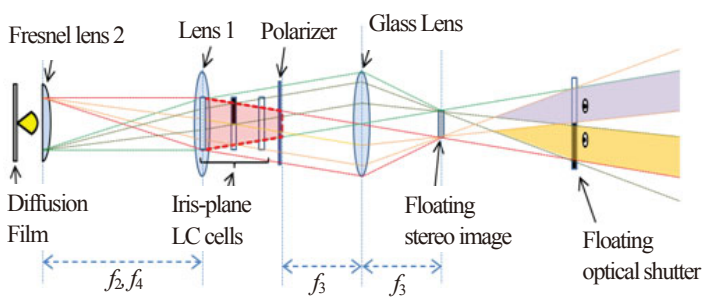

(b)

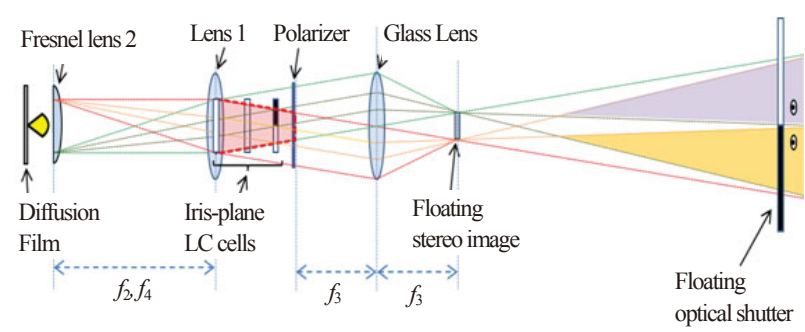

(c)

Fig.15 Depth control for the observation area by stacked LC Cells in new optical configuration, (a) short distance, (b) middle distance, and (c) long distance.

In addition, since the collimated image light passes through glass lens, we can suppress the image shift and image distortion due to the spherical aberration of glass lens. Fig. 14 shows the floating images for left and right eyes in our new optical system. The image distortion was suppressed compared to the previous configuration shown in Fig. 9. Further improvement will be realized when we use the aspherical lens in our optical system. 


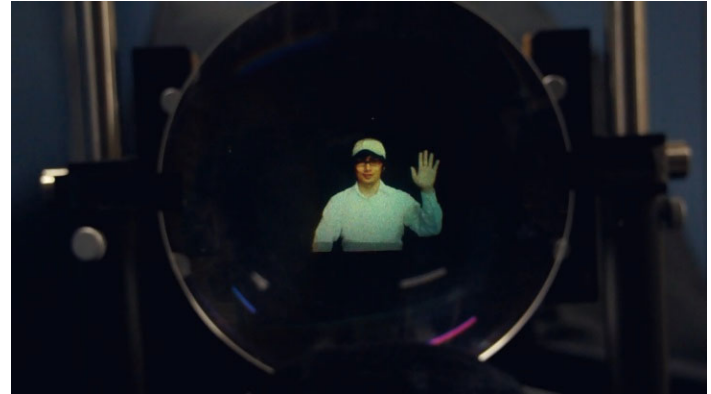

Fig.16 Displayed 3D image on the floating autostereoscopic 3D display system.

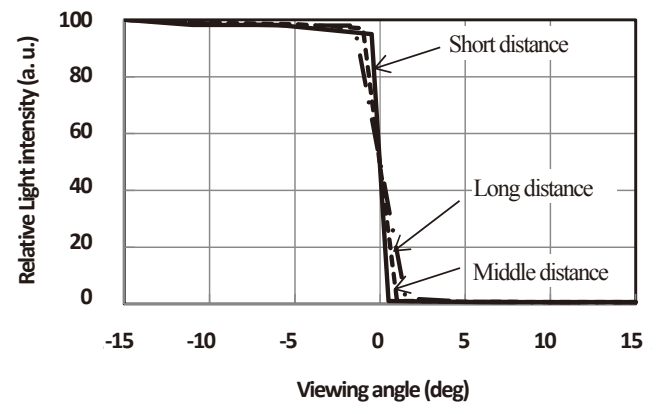

Fig.17 Luminance of 3D display system when scanning horizontally across the viewing zones for short, middle and long distances.

Fig. 15 shows the shift of the viewing zone toward the position of the liquid crystal cell. The focal length of Lens 1 is $400 \mathrm{~mm}$ and we used three LC cells. The distance of the LC cells from Lens 1 are $0 \mathrm{~mm}, 80 \mathrm{~mm}$ and $160 \mathrm{~mm}$ and viewing distance are $400 \mathrm{~mm}, 530 \mathrm{~mm}$ and $1000 \mathrm{~mm}$ respectively.

Fig. 16 shows displayed 3D images from our floating 3D display and Fig. 17 shows the measurement result of the luminance scanning horizontally across the viewing zones in several viewing distance. Here, the image for the right eye was in the black state and the image for the left eye was in the white state.

The luminance was uniform in the viewing zone and changed steeply at the boundary between the left and right viewing zones in any viewing distance.

Therefore, we confirmed that our display system produced high-resolution floating $3 \mathrm{D}$ images without crosstalk noise, high luminance uniformity and wide viewing zone depth.

\section{Conclusion}

We have developed the high-resolution floating autostereoscopic 3D display system with high light efficiency and wide viewing depth based on the irisplane dividing technology.

We electrically controlled the viewing zone discretely in the direction of the depth by changing the position of activated LC cell according to the visual distance and realized wide viewing depth. We also developed the light diffusion film which maintains the polarization state and has a top-hat light diffusing distribution, and realized high light efficiency and high luminance uniformity.

We confirmed that that our floating 3D display successfully produced floating stereoscopic images in the real space with high resolution, high light efficiency and no cross-talk noise in wide viewing depth. Therefore, we can observe highly realistic images regardless of the viewing distance, so this $3 \mathrm{D}$ display is promising for the future high-presence interactive communication systems. Realization of the distance control of the floating image according to the image contents will be a future subject for this system.

\section{Acknowledgement}

Supported in part by Konica Minolta Science and Technology Foundation Konica Minolta Imaging Science Award.

\section{References}

1) D.-S. Kim and S. Chestak: "Development of high resolution stereoscopic display based on LCD panel", Proc. of IDW '07, pp.2227-2230 (2007)

2) W.L. Hsu, C.S. Wu, L.C. Lin, C.L. Wu, C.H. Tsai and Kuen Lee: "A Viewer-Tracking-Based 2D/3D Switchable Autostereoscopic Display with Motion Parallax", Proc. of IDW '07, pp.2235-2238 (2007)

3) N.A. Dodgson, J.R. Moore, S.R. Lang: "Multi-view autostereoscopic 3D display", IBC '99 (International Broadcasting Convention) pp.497-502 (1999)

4) S. Pastoor and C. Conomis: "Mixed Reality Displays", 3D Video communication: John Wiley, pp.261-280 (2005)

5) N.A. Dodgson, J.R. Moore, S.R. Lang, G. Martin, P. Canepa: "A 50" time-multiplexed autostereoscopic display", Proc. of SPIE 3957, 177-83 (2000)

6) A.R.L. Travis, S.R. Lang, J.R. Moore and N.A. Dodgson: "Timemultiplexed three-dimensional video display", Journal of the SID, 3/4 pp.203-205 (1995)

7) H. Kakeya and Y. Arakawa: "Optical Design of Autostereoscopic Display with Real-Image Screen", Proc. of 3D Image Conference, pp.63-66 (2000)

8) H. Kakeya, M. Isogai, K. Suzuki and Y. Arakawa: "Autostereoscopic 3D Workbench", SIGGRAPH 2000 Conference Abstract and Applications, p. 78 (2000)

9) H. Kakeya and Y. Arakawa: "Autostereoscopic Display with RealImage Screen", SIGGRAPH 2000 Conference Abstract and Applications, p. 178 (2000)

10) H. Kakeya: "Real-Image-Based Autostereoscopic Display Using LCD, Mirrors and Lenses", Proc. of SPIE 5006, pp.99-108 (2003)

11) T. Ishinabe, T. Kawakami, N. Takahashi and T. Uchida: "Highresolution autostereoscopic 3-D projection display with a spacedividing iris-plane shutter", Journal of the Society for Information Display, 18, 8, pp.583-588 (2010)

12) T. Ishinabe, T. Kawakami and T. Uchida: "High-Resolution Floating Autostereoscopic 3D Display Based on Iris-PlaneDividing Technology", 2012 SID International Symposium Digest, 
pp.225-228 (2012)

13) H. Kakeya: "Autostereoscopic display with real-image virtual screen and light filters", SPIE Proceedings, 4660: Stereoscopic Displays and Virtual Reality Systems IX, pp.349-357 (2002)

14) H. Kakeya, N. Kobe, H. Kasano: "Multiview Autosterescopic Display with Floating Real Image", Proc. of SPIE-IS\&T, 5291 pp.255-264 (2004)

15) M. Nishizawa, K. Kusama, K. Sekiya, B. Katagiri, T. Kawakami, T. Uchida: "Investigation of Novel Diffuser Films for 2D LightDistribution Control", Pro. of the 18th International Display Workshops, pp.1385-1388 (2011)

16) M. Nishizawa, K. Sekiya, T. Kawakami, T. UCHIDA: "Synthesis and Optical Property Analysis of Novel Anisotropic Light Diffusing Film", Bulletin of the Chemical Society of Japan, 85, pp.839-841 (2012)

17) M. Nishizawa, K. Kusama, K. Sekiya, B. Katagiri, T. Kawakami, T. UCHIDA: "Novel Diffuser Films for 2D Light-Distribution Control", ITE Technical Report, 36, 11, pp.9-15 (2012)

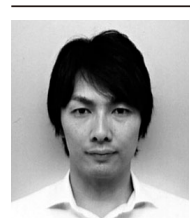

Takahiro Ishinabe received his B.S., M.S., and $\mathrm{Ph}$. D. degrees in Electronic Engineering from Tohoku University, Sendai, Japan, in 1995, 1997 and 2000 , respectively. From 2000 to 2002 , he was a Research Fellow of the Japan Society for the Promotion of Science and since 2003, he has been an Assistant Professor in the Department of Electronics, Graduate school of Engineering, Tohoku University. He has also been a Visiting Professor in the CREOL, The College of Optics and Photonics, University of Central Florida since 2010. He has been performing a research on advanced liquid crystal displays such as wide viewing angle LCD, reflective full-color LCD and field sequential color LCD. He received the Best Poster Paper Award from SID in 1998, the Outstanding Poster Paper Award from IDW in 2005, 2006, 2007, 2009 and in 2011, respectively, the Special Recognition Award from SID in 2011, Funai Award from Funai Foundation for Information Technology (FFIT) in 2012 and Fujio Frontier Award from ITE in 2013. He has been served as a Program Chairman of the workshop on LC Science \& Technologies at IDW since 2011. He is a senior member of Society for Information Display, a member of Japanese Liquid Crystal Society, Japan Society of Applied Physics and the Institute of Image Information and Television Engineers of Japan.

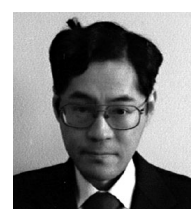

Tohru Kawakami received his B. S., M.S., and $\mathrm{Ph}$. D. degrees in Electronic Engineering from Tohoku University, Sendai, Japan, in 1991, 1993 and 1996, respectively. From 1996 to 2005 he was Research Associate, from 2005 to 2008 Lecturer, and since 2008 he has been Guest Associate Professor in New Industry Creation Hatchery Center, Tohoku University. He has been performing a research on optical computing and projection display system. He is a member of SID, The Institute of Image Information and Television Engineers of Japan, the Japan Society of Applied Physics, and the Laser Society of Japan.

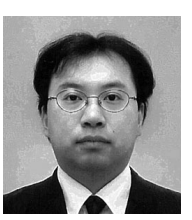

Masahiro Nishizawa received his B.S., M.S., and Ph. D. degrees in Science from Osaka City University, Osaka, Japan, in 1995, 1996 and 2000, respectively. In 2000 , he was a researcher in the National Institute of Radiological Science. From 2001 to 2003, he was an Assistant Professor in Kochi University of Technology and from 2003 to 2006, an Assistant Professor in Tohoku University. From 2006 to 2008, he was a researcher in New Energy and Industrial Technology Development Organization. Since 2008, he returned to Tohoku University as an Associate Professor. He has been performing researches on advanced functional materials such as light controlling polymers, electromagnetic organic compounds and pharmacological-activity compounds. In the field of the Display, he received the Outstanding Poster Paper Award from IDW in 2011.

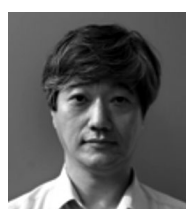

Hideo Fujikake received the B.E., M.E and Ph.D. degrees from Tohoku University, Japan, in 1983, 1985 and 2003, respectively. In 1985, he joined Japan Broadcasting Corporation (NHK). In 1988-2012, he was with NHK Science and Technology Research Laboratories (in 2002-2012, as a senior research engineer), engaged in the research and development of liquid crystal and organic materials/devices for various displays, optical filters and television program production equipment. He received Niwa-Takayanagi Best Paper Awards from ITE in 2003 and 2009, Technical Progress and Development Award from ITE in 2003, and Best Paper Award from IEICE in 2001. He was a visiting professor at Tokyo University of Science in 2006-2012. Since 2012, he has been a professor at Department of Electronic Engineering, Tohoku University. His current interest is concerned with flexible/printable electronics including flexible liquid crystal displays with high image quality. He also served as a member in Director Board of Japanese Liquid Crystal Society in 2004-2005 and 2011-2012, and a chairman at Technical Committee on Electronic Information Displays of IEICE in 2012. He is now a chairman at Technical Group on Information Display of ITE, a workshop chair on Flexible Displays in International Display Workshops (IDW), and a chair at Japan Chapter in IEEE Consumer Electronics Society.

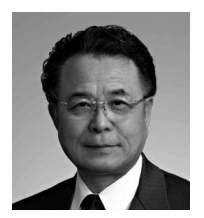

Tatsuo Uchida received his BS, MS and Ph.D. degrees in electronic engineering from Tohoku University in 1970, 1972 and 1975, respectively. From 1975 to 1982 he was Research Associate, from 1982 to 1989 Associate Professor, from 1989 to 2010 Professor in the Department of Electronic Engineering, Tohoku University, and from 2006 to 2009 Dean of Faculty of Engineering and Graduate School of Engineering, Tohoku University. Since 2010 he has been President of Sendai National College of Technology. He received more than twenty awards for his contribution to science and technology on the liquid crystal display from the Director of the Science and Technology Agency, the Minister of Education, Culture, Sports, Science and Technology, JLCS (the Japanese Liquid Crystal Society), SID (Society for Information Display), IEICE (The Institute of Electronics, Information and Communication Engineers), ITE (The Institute of Image Information and Television Engineers), etc. Prof. Uchida is Fellow of SID, IEICE and ITE. He has been engaged in organizing many conferences including the Japan Display '92 and IDW '97 as Program Chair, the International Liquid Crystal Conference 2000 as Executives Chair, and IDW '03 and IDW '04 as General Chair. He was also President of Japanese Liquid Crystal Society, and President of ITE. 